\title{
A Generalized Model for Fuel Channel Bore Estimation in AGR Cores
}

\author{
Andrew Young ${ }^{a, *}$, Craig Berry ${ }^{\mathrm{a}}$, Graeme M. West ${ }^{\mathrm{a}}$, Stephen D.J. McArthur ${ }^{\mathrm{a}}$ \\ ${ }^{a}$ Institute for Energy and Environment, Department of Electronic and Electrical Engineering, University of Strathclyde, 204 \\ George Street, Glasgow, G1 $1 X W$, U.K.
}

\begin{abstract}
One of the major life-limiting factors of an Advanced Gas-cooled Reactor (AGR) nuclear power station is the graphite core as it cannot be repaired or replaced and therefore detailed information about the health of the core is vital for continued safe operation. The graphite bricks that comprise the core experience gradual degradation during operation as a result of irradiation. Routine physical inspection of the graphite core fuel channels is performed by specialist inspection equipment during outages every 12 months to 3 years. It has also been shown to be advantageous to supplement this periodic inspection information with analysis of operational data which can provide additional insights into the core health. One such approach is through the use of online monitoring data called the Fuel Grab Load Trace (FGLT). An FGLT is a measure of the perceived load of the fuel assembly with contributions from aerodynamic forces and frictional forces, which is related to bore diameter.

This paper describes enhancements to existing analysis of FGLT data which, to date, has focussed solely on using data from a single reactor at a time to build bore estimation models, by considering data from multiple reactors to produce a generalised model of bore estimation. This paper initially describes the process of producing a bore estimation from an FGLT by isolating the contribution that relates to the fuel channel bore and then discusses the limitations with the existing bore estimation model. Improvements are then proposed for the bore estimation model and a detailed assessment is undertaken to understand the effect of each of these proposed improvements. In addition, the effect of introducing non-linear regression models to further enhance the bore estimation is explored. The existing model is trained on data from one reactor in the UK and therefore the results produced from it are only applicable to this reactor. However, out of the remaining 13 nuclear reactors currently in operation, 3 also have a similar construction to the reactor the model is trained on, and these should all produce similar FGLT data. Therefore, a generalised model is proposed that produces bore estimations for four AGRs stations reactors, compared with one previously. It is shown that this approach offers an improved overall bore estimation model.
\end{abstract}

Keywords: Fuel grab load trace, condition monitoring, advanced gas-cooled reactor (AGR), graphite core, nuclear engineering, nuclear power plant

\section{Introduction}

Currently, there are seven advanced gas-cooled reactor (AGR) power stations operating in the United Kingdom, each with two identical reactors and all operated by EDF Energy (World Nuclear Association (2017)). These 7 stations are all approaching their planned closure date (see Table 1). The graphite bricks that make up the core act as

\footnotetext{
*Corresponding author. Tel.: 01414447241

Email address: andrew.young.101@strath.ac.uk (Andrew Young)
}

both a moderator in the nuclear reaction and also 10 provide structural integrity for the entire core. As these bricks cannot be repaired or replaced they are one of the main life-limiting factors of these type of power stations.

Therefore a significant element of the decisionmaking process on whether to extend the lifetime of these stations is the routine assessment of the health of the graphite core. This can be done in one of two ways, firstly through physical inspection during outages that occur every 12 months to 3 20 years and secondly through monitoring data gath- 


\begin{tabular}{l|c|c|c|c} 
Station & $\begin{array}{c}\text { Construction } \\
\text { Started }\end{array}$ & $\begin{array}{c}\text { Connected } \\
\text { to grid }\end{array}$ & $\begin{array}{c}\text { Original } \\
\text { closure date }\end{array}$ & $\begin{array}{c}\text { Extended } \\
\text { closure date }\end{array}$ \\
\hline Dungeness B & 1965 & 1983 & 2018 & 2028 \\
Hartlepool & 1968 & 1983 & 2018 & 2024 \\
Heysham 1 & 1970 & 1983 & 2018 & 2024 \\
Heysham 2 & 1980 & 1988 & 2023 & 2030 \\
Hinkley Point B & 1967 & 1976 & 2011 & 2023 \\
Hunterston B & 1967 & 1976 & 2011 & 2023 \\
Torness & 1980 & 1988 & 2023 & 2030
\end{tabular}

Table 1: Construction, connection, original closure and extended closure dates of all 7 AGRs currently in operation World Nuclear Association (2017)

ered during refuelling events, which occur every 6 to 8 weeks. The physical inspection and video inspection footage provides very accurate measurements of the fuel channel, however, as the power plant is required to be shut down to perform this the data is gathered periodically and monitoring data is used to give more continual and frequent assessments of the core state. One type of monitoring data used to achieve this is fuel grab load trace (FGLT) data, 30 which was originally used for safety purposes to protect the reactor in the event of any faults during refuelling. The data retrieved is a measure of the perceived weight of the fuel assembly as it is being removed or inserted into the core.

35 From previous work, it has been shown that this FGLT data can be used to provide information about the variations in the diameter of the fuel channel bore. In West et al. (2006, 2007) an introduction to the concept of FGLT analysis was dis40 cussed as well as a thorough overview of the data capture system. West et al. (2009) discusses the British Energy Trace Analysis (BETA) system, an automated intelligent analysis system for the assessment of FGLT data. The concept of isolating and 45 removing various components of the FGLT data was discussed in West et al. (2014) and using the corresponding inspection data previously gathered it was shown that it is possible to train a predictive model to estimate the fuel channel bore diameter ${ }_{50}$ and thus the health of the core from each individual FGLT. Berry et al. (2017) expands upon this by proposing improvements to the model which increases the accuracy in the upper core regions as well as providing estimations for a larger area of 5 the fuel channel. The work discussed in Berry et al. (2017) focused on providing bore estimations for offload depressurised refuelling data, Young et al. (2018) expanded on this by producing estimations for onload pressurised refuelling data by removing 60 the aerodynamic effects. To date all this work has focused on data gathered from a single reactor, the novel contribution of this paper is the generalization of fuel channel estimation to a single model trained on data from multiple reactors at different operational stages, with different histories.

The next section of this paper provides background information about the construction and refuelling of AGR cores and also the various defects that can occur during operation. Details of the differences between the FGLT data and the diameter measurements taken during inspection are presented in the next section. Section three presents a breakdown of the various components of an FGLT, while section four presents the existing bore estimation model along with some proposed changes to improve the implementation. Section five presents the results and a comparison between different versions of the bore estimation model and section six proposes a generalised bore estimation model to o produced bore estimates for two stations, four reactors, in the UK. Finally, the conclusions and future work are presented in section seven.

\section{Advanced Gas-cooled Reactors}

\subsection{Overview}

Out of the eight nuclear power stations in the UK currently in operation, seven of these are AGR design, the other Sizewell B is a pressurized water reactor (World Nuclear Association (2017)). An AGR core is made from thousands of interlocking hollow cylindrical graphite bricks. Fig. 1a and 1b, show a small section of a core and the arrangement of the graphite bricks within it. Graphite not only provides structural integrity to the core but also acts as the moderator (Bradford and Steer (2008)). 


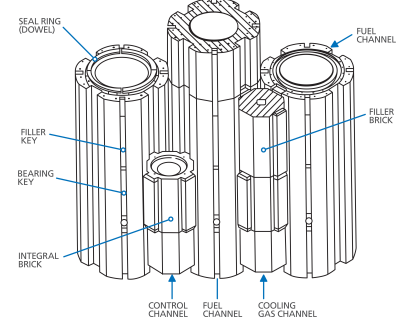

(a)

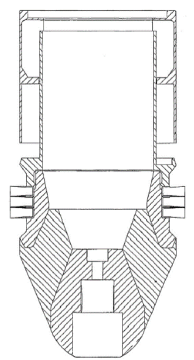

(c)

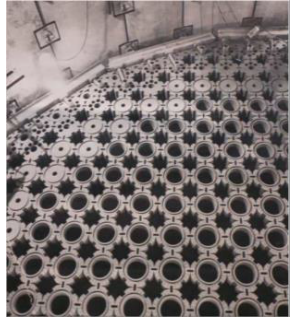

(b)

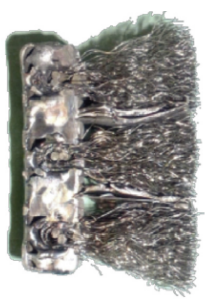

(d)
Figure 1: (a) Graphite brick arrangment within an AGR core (b) Graphite core during construction, (c) Fuel stringer nose assembly, and (d) Lower stabilisng brush (Steer (2007))

The vertical fuel, control and cooling gas channels, typically are composed of a stack of eleven graphite bricks and allow for the insertion of the fuel assembly (Fig. 2), control rods and the circulation of the carbon dioxide coolant respectively.

As the graphite is irradiated in the reactor it begins to shrink, however, in later life it begins to expand (Brocklehurst and Kelly (1993); Marsden et al. (2008); Crump et al. (2017); Maul et al. (2017)), the point that this occurs is called the graphite turnaround point. The shrinkage or expansion of the graphite has a resulting effect on the fuel channel bore diameter. Further to this at the turnaround point the internal stresses on the graphite brick reverse, at some point after this time a crack can occur axially which can also increase the diameter of the fuel channel. In an attempt to replicate this effect, experiments have been performed (Bradford and Steer (2008)) which have produced secondary cracks in the test graphite bricks. If this to happen in the AGR core it could also have the effect of reducing the diameter of the fuel channel bore. This could potentially lead to issues with the insertion or removal of the fuel from the fuel channels.
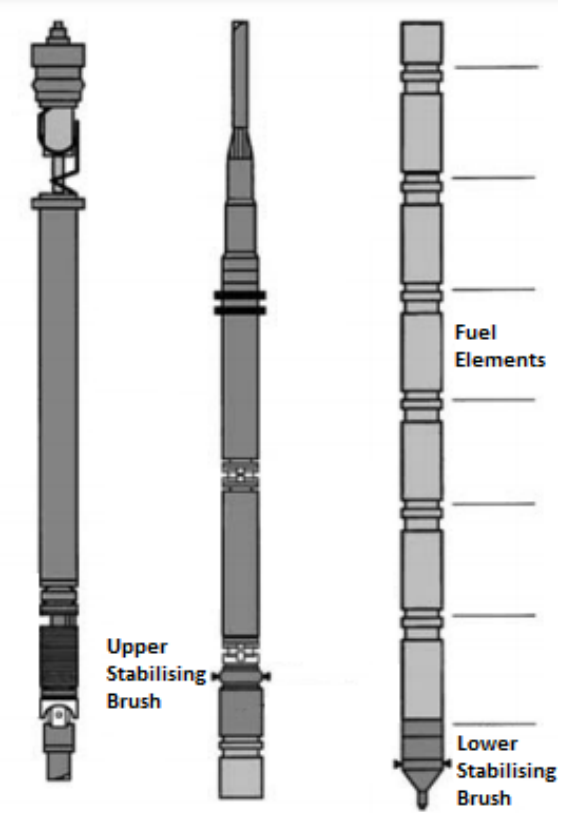

Figure 2: Fuel assembly (Pang et al. (2007))

\subsection{Condition Monitoring and Inspection}

Every 12 months to 3 years during planned outages inspection of the AGR cores is performed. During this time the bore diameter of the fuel channel bricks is measured either by a Channel Bore 125 Inspection Unit (CBIU) or a New In Core Inspection Equipment mark 2 (NICIE 2) Cole-Baker and Reed (2007)). Both of these devices perform a physical inspection of the fuel channel and can, therefore, be considered as accurate. Fig. 3a shows an example of inspection data retrieved during one of these planned outages. To safely determine the health of the core up to 31 out of 308 channels are inspected at any of these outages, however, this number has increased as the reactors have approached the end of their design lifetime. As the inspection data can only be gathered when the reactor is offline and is therefore performed periodically,

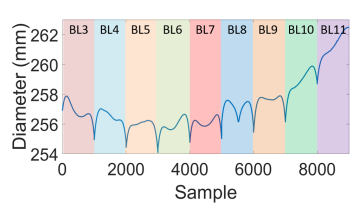

(a)

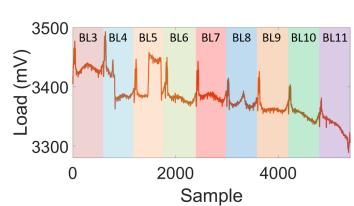

(b)
Figure 3: Example of (a) inspection and (b) monitoring data for brick layers 3 to 11 of an AGR core 
rather than continuously, any additional information that can be gained during normal operation eneficial and can be used to support the safety case. One way of achieving this is by using monitoring data gathered during refuelling events that occur every 6 to 8 weeks. This data was originally only used to inform the reactor protection trip sysfuelling. As the fuel stringer is inserted or removed from the fuel channel, load cells on the refuelling machine directly measure the perceived load of the fuel assembly in millivolts. This perceived load will be a combination of the frictional and aerodynamic forces acting on the fuel assembly, by isolating the frictional component, which is related to bore diameter, a better understanding of the health of the fuel channel can be achieved. Fig. 3b shows an example of the FGLT data recorded during a refuelling event. This FGLT data is an indirect measure of bore diameter and contains contributions from other effects so is far less accurate than the inspection data. Further processing of the data is also required before a bore estimation can be retrieved.

This section has presented an overview of the AGR system, the evolution of the graphite core during operation, and also discussed the current monitoring and inspection equipment. A thorough examination of all aspects of the AGR stations is beyond the scope of this paper. However, further information and a more thorough background into the AGR design can be found in British Electricity International (1992).

\section{Fuel Grab Load Trace Components}

Previous work in West et al. (2014); Berry et al. (2017); Young et al. (2018) has shown that an FGLT can be modelled as a combination of four components. These four components are the contribution from the lower $\left(K_{L}\right)$ and upper $\left(K_{U}\right)$ stabilizing brushes, the deadweight of the fuel stringer $(m)$, and the aerodynamic effects $\left(F_{G}\right)$ due to gases in the fuel channel. Through discussion with specialist engineers, theoretical understanding and extensive FGLT data exploration the approximate contributions for each of these components are shown in Fig. 4.

Eq. 11 (Berry et al. (2017) ) shows the corresponding load value in an FGLT given the height $(h)$ of 185 the lower stabilising brush (LSB) in the fuel chan-

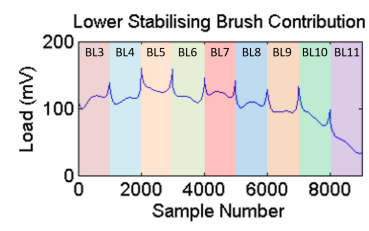

(a)

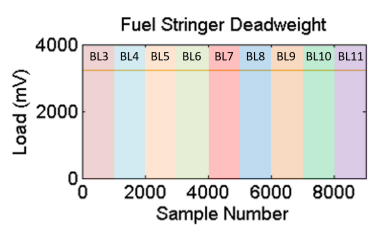

(c)

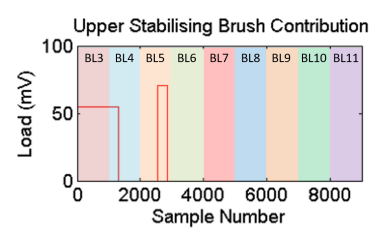

(b)

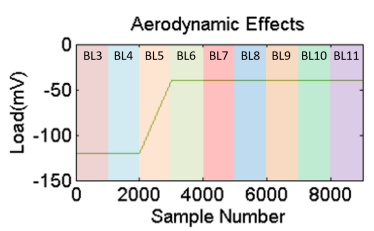

(d)
Figure 4: Approximated components of an FGLT (a) Lower stabilising brush contribution (b) Upper stabilising brush contribution (c) Fuel stringer deadweight (d) Estimation of aerodynamic effects

nel:

$$
L(h)=K_{L}(\Phi(h))+K_{U}(\Phi(h+d))+F_{G}(h)+m(1)
$$

where $K_{L}(\Phi(h))$ is the LSB frictional component for the bore diameter $(\Phi)$ at height $h, K_{U}(\Phi(h+$ $d))$ is the upper stabilising brush (USB) frictional component at height $(h+d)$ where $d$ is the distance between the lower and upper stabilizing brushes, $F_{G}(h)$ is the aerodynamic forces which change with height $(h)$, and $m$ is the mass, or deadweight, of the fuel stringer $(m)$ which is constant throughout each trace (West et al. (2014)).

The approximate LSB contribution $\left(K_{L}(\Phi(h))\right)$ is shown in Fig. 4a. This contribution is the component that is of most interest as this contribution is related to the fuel channel bore diameter. Fig. $4 \mathrm{~b}$ shows the approximate contribution from the upper stabilizing brush (USB) $\left(K_{U}(\Phi(h+d))\right)$. For

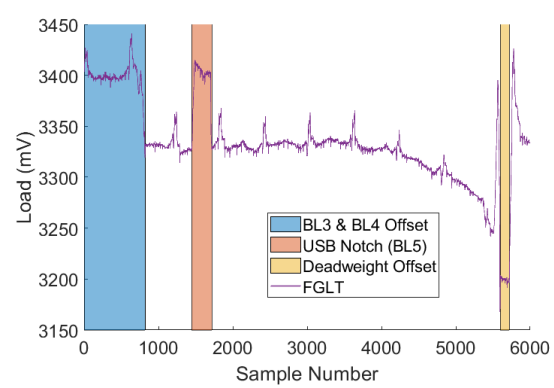

Figure 5: Example of FGLT data, with USB contributions and fuel stringer deadweight highlighted 


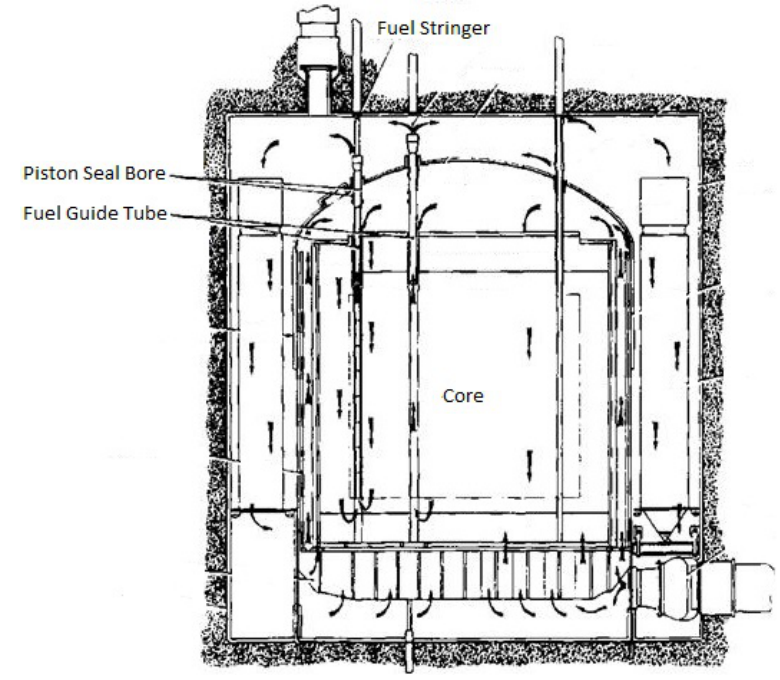

Figure 6: Diagram of AGR core, with piston seal bore and fuel guide tube highlighted British Electricity International (1992)

the majority of the discharge or charge, the USB does not have an effect on the FGLT due to havng a smaller diameter than the LSB. However, at two points in the FGLT, see Fig. 5, there is a frictional contribution from the USB. The first point is when the USB passes through the start of the guide tube (Fig. 6), this occurs from the start of refuelling and first appears in the FGLT when the LSB is passing through brick layer 3 and ends partly through brick layer 4 . This is noticeable in the corresponding FGLT as an offset throughout this region. The second point is when the USB passes a piston seal bore (Fig. 6), this is when the LSB is passing through the middle of brick layer 5 and is noticeable as a step change in this region. Fig. $4 \mathrm{c}$ is the deadweight of the fuel stringer $(\mathrm{m})$. The mass of the fuel assembly is slightly different for each refuelling, depending on whether new or rotated fuel is being inserted, and thus has the effect of a different constant offset in the FGLT. It is however assumed that the mass will not change throughout the course of each individual refuelling. The last component, which is also the most complex as the effects cannot be easily modelled Young et al. (2018), relates to the various aerodynamic effects caused by gas circulating throughout the core supporting some of the weight of the fuel assembly. This can be broken down into three regions; an in core region, an out of core region and a transitional region between the two. Fig. $4 \mathrm{~d}$ shows an estimation of the aerodynamic effects $\left(F_{G}(h)\right)$. The removal of these gas effects is beyond the scope of 235 this paper, and hence the rest of the paper is only applicable for offload depressurised refuelling where the gas effects are negligible. Young et al. (2018) discusses an approach for the removal of the gas effects from onload pressurised data.

\section{4. Bore Estimation Model}

\subsection{Existing Model}

The bore estimation algorithm presented in Berry et al. (2017) improved the accuracy of estimating the fuel channel bore diameter from an 245 FGLT compared with the previous iteration presented in West et al. (2014). It was shown through iterative improvements and multiple models that the root mean square error and maximum error was improved, this was especially notable in brick layers

2507 to 10 . Another major improvement over the previous version was the area covered by the estimation which included previously neglected regions in the previous models (brick layers 3 to 5 ). These were originally neglected as there was no pre-processing step to remove the USB contributions in the initial model.
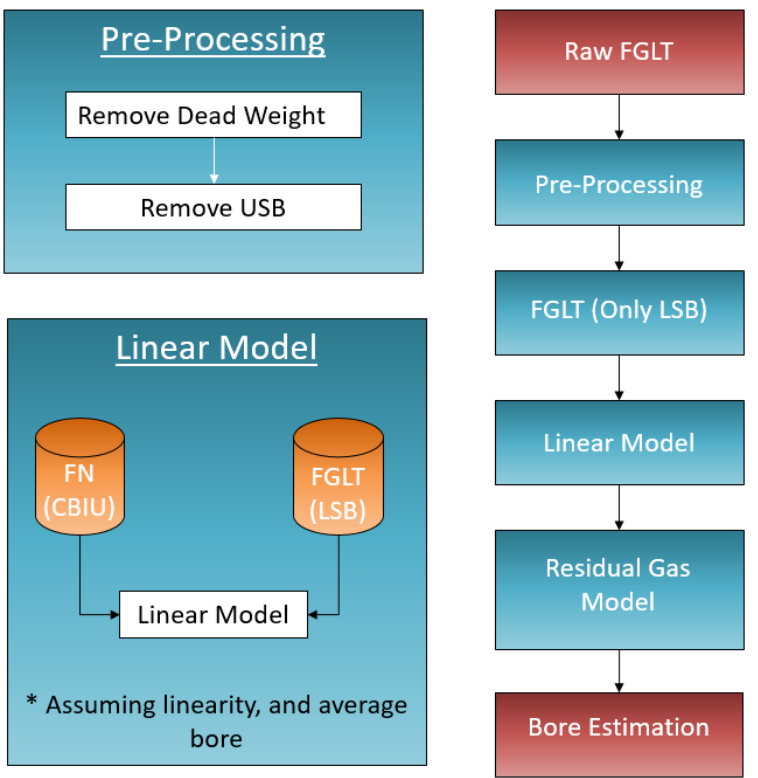

Figure 7: Bore estimation model process from Berry et al. (2017)

Fig. 7] shows the existing process of producing a bore estimation from a raw FGLT. The initial 


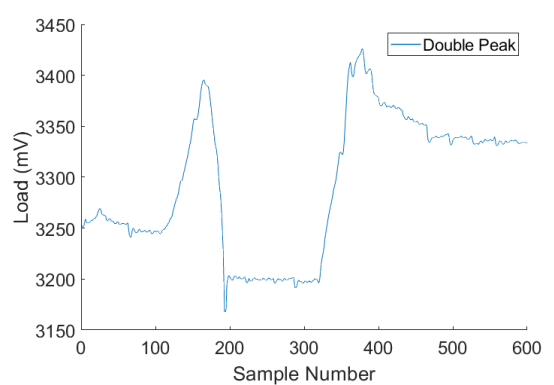

Figure 8: Example of data from double peak region

step is to perform pre-processing on the data, to atthe additional components of the FGLT (Fig. 4). Firstly, the deadweight of the fuel stringer is estimated by examining the load value for a region in the FGLT where neither the LSB or USB has any frictional contribution, and the gas efare negligible. This occurs directly after brick layer 11 when the LSB is passing through the piston 290 seal bore and is called the double peak region (Fig. 8). Currently, the load value is visually determined then entered manually into the algorithm. The secd pre-processing step is to remove the USB contributions, as these always occur in three regions: 295 brick layer 3 and the start of brick layer 4 (Fig. 9 and the middle of brick layer 5 (Fig. 10). An algorithm was designed to detect the step change at the start of brick layer 4, the end of the first USB contribution region, and also both step changes at 300 either side of the USB notch. By assuming these contributions are both constant these effects can be removed. The constant offset was determined by taking the average load value for the USB notch region in brick layer 5. However, this introduced 305 a slight error at the transition regions of the USB notch, see Fig. 11c, this was visible as a transient

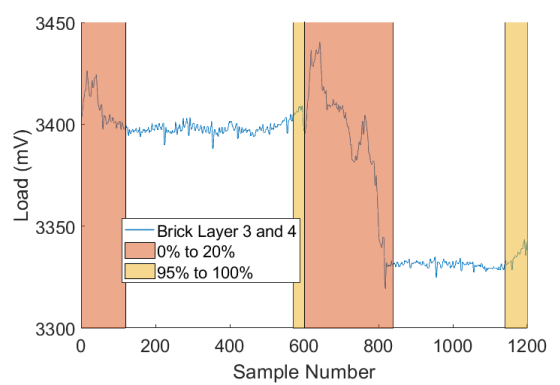

Figure 9: Example of data from brick layer 3 and 4, with excluded regions highlighted response between the different load values. Having 285 performed these pre-processing steps the resulting load should be only the contribution from the LSB.

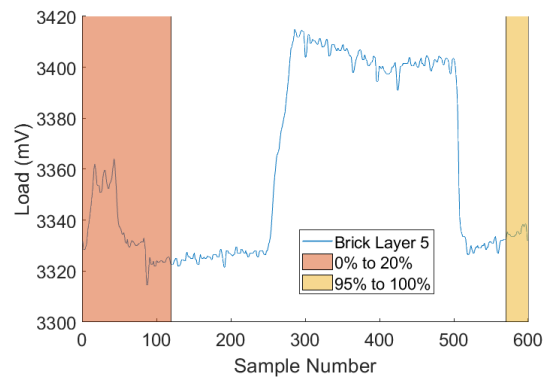

Figure 10: Example of data from brick layer 5, with excluded regions highlighted

The next stage was to train a linear regression model to predict bore estimations from FGLT data. Only brick layers 6 to 11 are used in the training process, as these brick layers contain no additional contributions to the FGLT other than the LSB. Due to there being three sets of brushes on the fuel assembly, see Fig. 1c and 1d, the data for any specific brick layer contains data relating to the transition region which occurs over the first $20 \%$ of the data, see Fig. 9 and 10 . Likewise, the end of the data contains the beginning of the transition region which covers the final $5 \%$ of the data. Therefore, only the data from $20 \%$ to $95 \%$ of each brick layer was used for training as the interaction of the LSB at the brick interfaces is different than with the fuel channel walls. The linear model was trained using 20 sets of CBIU and corresponding FGLT data, from various channels for one specific reactor.

In an attempt to remove any residual gas effects from the estimation a second non-linear regression model was produced after the inclusion of the data from brick layers 3 to 5 , which contain USB friction. By selecting a data point at the end of brick layer 10 and normalising each bore estimation to that point a relative estimation of the bore diameter can be produced. Therefore, rather than being a direct estimation of bore the existing model estimates how different the bore is compared with the that there was a gradual increase in the prediction error from brick layer 10 downwards that followed a non-linear relationship. A second order polynomial was trained to remove this effect, the assumption is made that the residual offset in the estimation is due to aerodynamic effects caused by the transition 


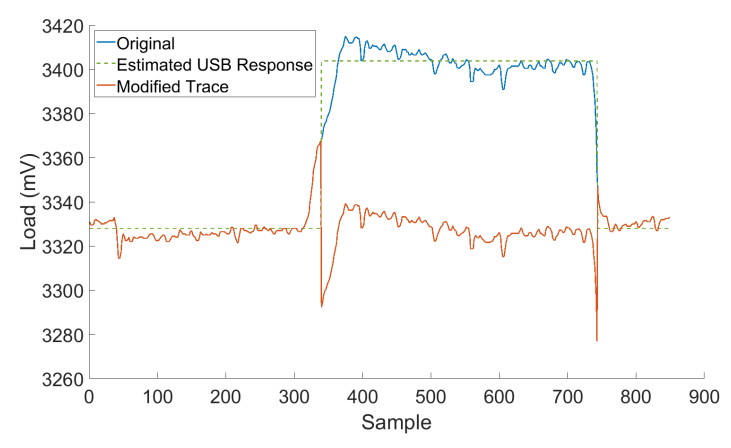

(a)

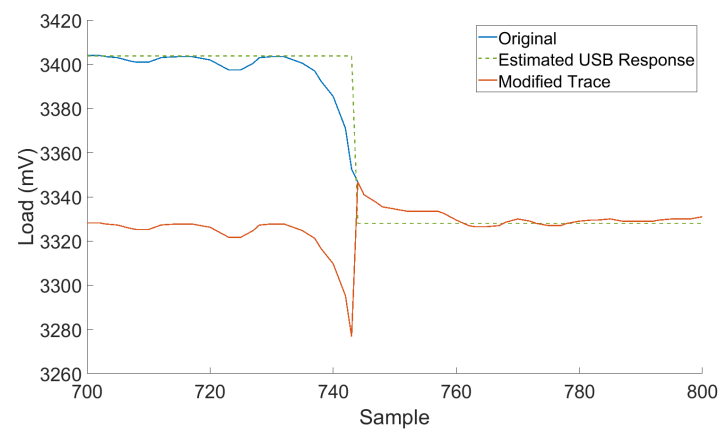

(c)

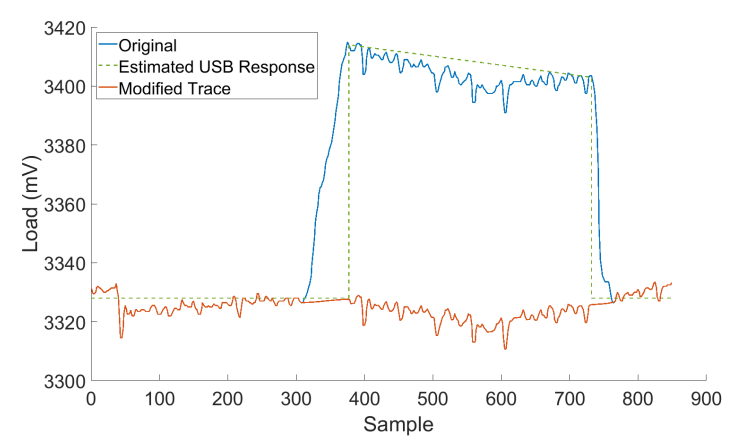

(b)

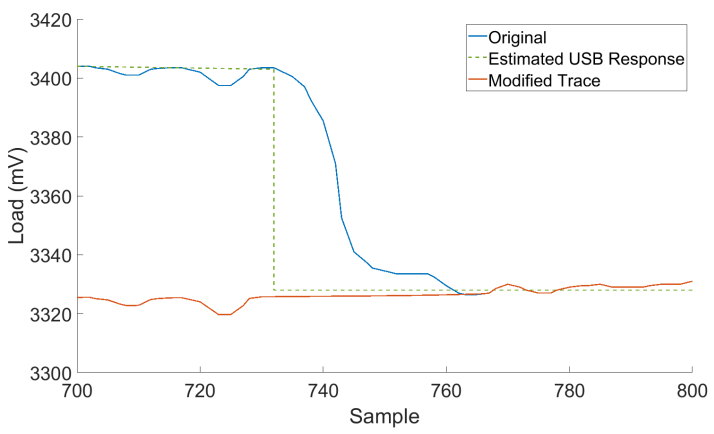

(d)

Figure 11: (a) Existing method from removing USB notch (b) New method from removing USB notch (c) Transition region removal using existing method (d) Transition region removal using new method

of the fuel assembly from inside to outside the core.

Eq. 2 shows the existing bore estimation model, 345 where $\Phi$ is the bore in mm for a specific height, h, in

the core. $K_{L}$ is a linear function that relates load to bore, $L$ is the load at a specific height, $K_{U}$ is the required offset to remove the USB interaction at a specific height, $K_{O}$ is the second polynomial 350 regression function at a specific height and $H_{O}$ is the required offset to normalise the bore to the end of brick layer 10 using historical data (Berry et al. (2017)).

$$
\Phi(h)=K_{L}(L(h))-K_{U}(h)+K_{0}(h)+H_{0}
$$

\subsection{New Model}

Several improvements have been made to the bore estimation model since the version detailed above. The changes that have been made to the pre-processing stage include the implementation of 360 a new technique to remove the USB notch in brick layer 5 that eliminates the transient response, the removal of the USB frictional component in brick layer 3 and brick layer 4 is now independent of the load value in brick layer 5 and the deadweight offset 365 is now retrieved without user input by calculating the value between the double peak region, see Fig. 5. The existing model produces a relative estimate for bore diameter as the data is normalised to the bore of brick layer 10, this has been removed in the proposed model in favour of an absolute estimate of the bore.

\subsubsection{Brick Layer 5}

In the previous iteration of the bore estimation model, the USB frictional contribution in brick layer 5 was removed by first finding the maximum 355 and minimum slope in brick layer 5 (Fig. 10). This produced two points in the centre of the ramp up and ramp down regions of the USB notch. In Fig. 11a and 11c where the dotted green line (Estimated USB Response) crosses the blue line (Original) is the two points that were detected as the centre of the ramp up and down region. The average load value between these two points was taken as the USB contribution to the FGLT, and this was removed as a constant offset from the trace, see Fig. 11a. The main two issues with this approach are 
that the load across the USB region is not always constant, it can increase or decrease based on other effects in the fuel channel and also as discussed ear- ${ }_{405}$ lier there is a transient response in the ramp up and ramp down regions. Using the same method as the existing technique the new approach determines the centre of the ramp up and ramp down regions, then looks forward and backwards to find the start and end points, the point where the derivative changes sign. The ratio between the load at the start and end points is calculated, and a line with constant gradient is produced to connect these two ratios. This line is then multiplied by the load value to re- ${ }_{415}$ move the effects of the USB, also the values between the start and end points on each side are interpolated to produce a complete trace, see Fig. 11b.

\subsubsection{Brick Layer 3 and 4}

Through exploration of the data, it was found that the original assumption in West et al. (2014) that the offset caused by the USB in brick layers 3 and 4 was equal to the offset in brick layer 5 was incorrect (Fig. 12). After analysis of the fuel channel schematics, this is believed to be due to the different diameter of the piston seal bore and guide tube (Fig. 6). Similar to the new approach for brick layer 5 , the start and end point of the ramp down region was found. In this case it was found that the load remains relatively constant throughout brick layer 3 and 4 , therefore the average load between the start of brick layer 3 and the start of the ramp down region was calculated and this was used as the offset to remove the effect of the USB in brick layers 3 and 4 . Also as before the transition region was interpolated to produce a complete trace.

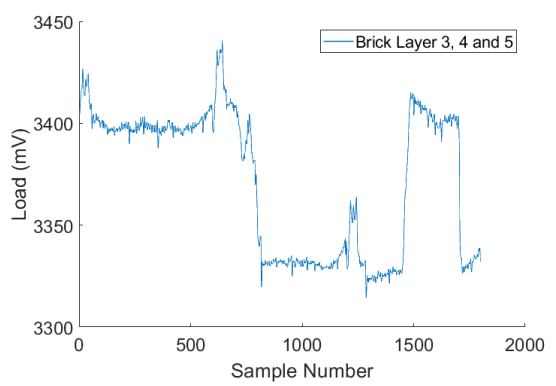

Figure 12: Example of data from brick layer 3 to 5

\subsection{Relative vs. Absolute}

Due to the existing model producing a relative measure for bore diameter, and also the dependency on historical data to perform the normalization, it is proposed that the slight improvement in accuracy of using a relative model is not worth the drawback of being reliant on historical data to perform the estimation. Therefore to move back to an absolute measure of bore diameter the linear model from the "First Bore Estimation Model" detailed in Berry et al. (2017) was used, however, the remaining improvements were still included. Also since that paper was published there is significantly more FGLT and CBIU data available. Using this a new 1st order polynomial (linear) model was trained using additional offload depressurised data from Reactor 1 (the same reactor as used previously).

\subsection{Non-Linear Model}

Experimental work by AMEC NNC Limited (Skelton (2007)) proposed that a linear model does not accurately represent the brush friction characteristics, and they produced a cubic model that fit the data from their experiments. In an attempt to integrate this into the proposed bore estimation algorithm, both a 2nd and 3rd order polynomial 25 (quadratic and cubic respectively) were trained using the same data used to train the new linear model previously. Due to the physical nature of the problem, that is the frictional forces are related to the diameter of the fuel channel. These forces will only follow the shape of a 2 nd or 3rd order polynomial over a specific range, see Fig. 13 data gathered out with the train data (Approx. 80 to $200 \mathrm{mV}$ ) may produce incorrect estimations.

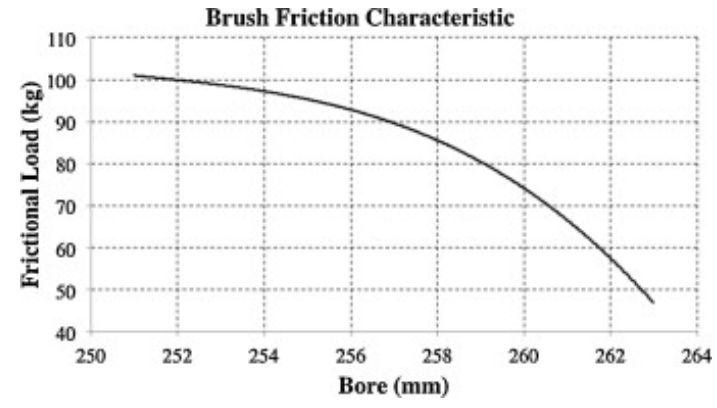

Figure 13: Plot of variation of brush friction load against bore obtained from experimentation (West et al. (2014))

\section{Initial Results}

\subsection{Training and Testing Data}

As the existing model was trained only on data from Reactor 1 before attempting to develop a generalized model it was necessary to acquire baseline 
results for the accuracy of each model version. Out corresponding inspection data, 25 were set aside for testing, while the other 25 were used to train the new linear, quadratic and cubic model for converting load to a bore estimate. For consistency, the three new models were trained using a least squares regression model over the same range as used previously, $20 \%$ to $95 \%$.

\subsection{Accuracy Metrics}

In order to determine the accuracy of each bore estimation model, it was necessary to select metrics that would be suitable for determining the overall accuracy of the bore estimation as well as the accuracy for each individual brick layer. The two metrics that were selected were the root mean square error (RMSE) and the maximum error (ME) in mil- 480 limetres. The RMSE is a metric that is often used to measure the difference between predicted values from a model and the observed values. The RMSE is the square root of the average of the squared residuals, where the residuals are the difference between predicted and observed values, see Eq. 3 . where, RMSE is the Root Mean Square Error, $n$ is the number of samples, $y$ is the CBIU data, and $\hat{y}$ is the bore estimation. Therefore, the effect of each error on the resulting error value is proportional to 490 the size of the squared error, making the RMSE very sensitive to outliers.

$$
R M S E=\sqrt{\frac{1}{n} \sum_{i=1}^{n}\left(y_{i}-\hat{y}_{i}\right)^{2}}
$$

The second metric selected was to take the maximum error in millimetres for the centre of each brick layer $(20 \%$ to $95 \%)$. Using both of the metrics selected, it should be possible to assign quantitative values to the accuracy of the bore estimations for each model.

\subsection{Results}

Having implemented all the changes detailed previously there were now five versions of the bore estimation model. Version 1.0 is the existing bore estimation model, version 1.1 is the existing bore estimation model, with improved implementation detailed in Sections 4.2.1 and 4.2.2 version 1.2 is the retrained linear model using the data from Reactor 1 as explained in Section 5.1, also with the improved implementation, version 1.3 is the trained quadratic model, with improved implementation, and version 1.4 is the trained cubic model, with improved implementation.

Using all five models discussed previously a bore estimation was produced for each of the 25 remaining FGLTs. Using the metrics detailed earlier a

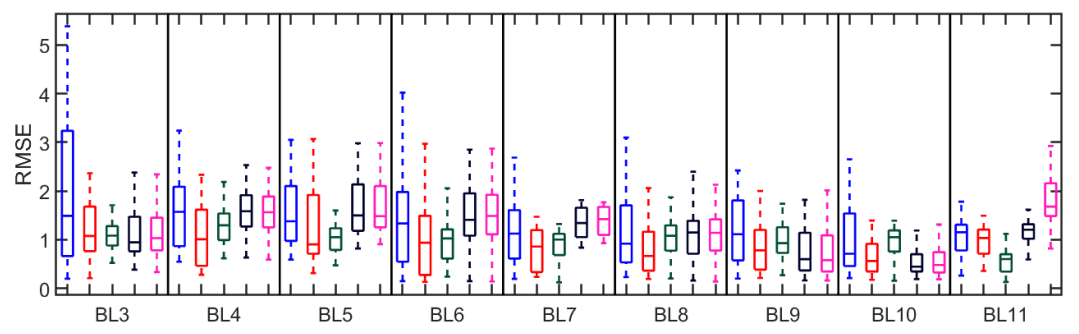

(a) Root mean square error for each individual brick layer in the test dataset

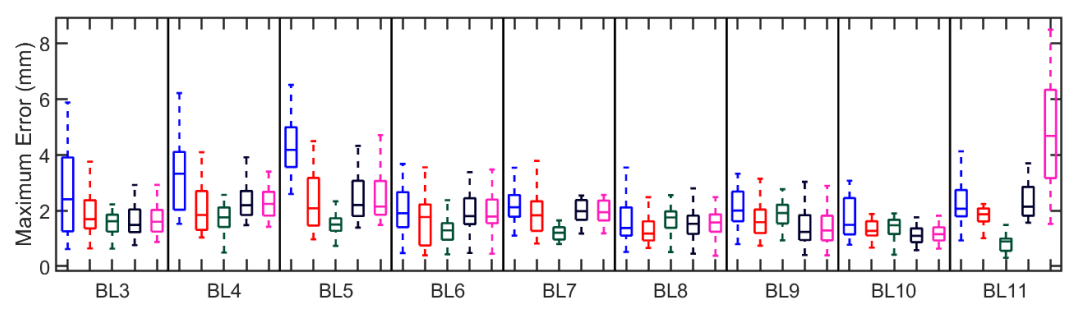

(b) Maximum error ( $\mathrm{mm}$ ) for each individual brick layer in the test dataset

Figure 14: Results for each model for all brick layers, (Blue) V1.0, (Red) V1.1, (Green) V1.2, (Black) V1.3, and (Pink) V1.4 


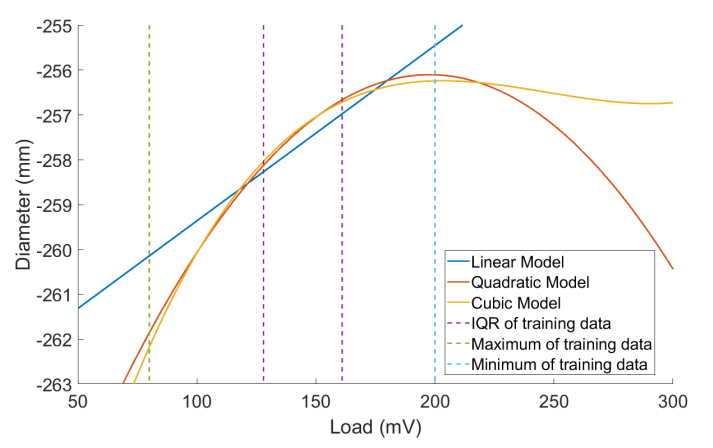

Figure 15: Linear, quadratic and cubic bore estimation models, with interquartile range, minimum and maximum of training data highlighted

value was produced for both metrics for each in- ${ }_{540}$ dividual brick layer in the FGLT. The values are shown for all FGLTs in Fig. 14.

From these results it is clear that for both metrics with the implementation changes alone there is a considerable improvement over the existing tech- 545 nique for both the RMSE and maximum error, this is especially noticeable in brick layer 5 and above. As expected, retraining the linear model with more data has improved the results dramatically, with the maximum error in brick layers 3 and 4 reducing ${ }_{550}$ by more than $1 \mathrm{~mm}$. Over the interquartile range of the training region the linear, quadratic and cubic models all produce very similar results, however, at the extremes the values produced for the quadratic and cubic models are unrealistic. When comparing the quadratic and cubic models to the linear model there is some improvement in brick layers 3 to 10 , however, the results for brick layer 11 are worse. As brick layer 11 is the least irradiated of all brick layers it should be the closest to start 560 of life diameter of $263 \mathrm{~mm}$. Because of this, there is also a lack of training data around this point, see Fig. 15. which results in the poor performance for both the quadratic and cubic models.

Based on these results it is clear that the best 565 model is version 1.20, the linear model, as this provides the best results for all the bricks covered in the FGLT. Therefore, it was decided that this should be used to design a generalized model for Reactors 1 to 4 . It should also be noted that if there was 570 more training data at the extremes then this assumption may not hold true, however, it is unlikely that this data will ever exist in the real world operation of AGRs. This is because the degradation of the graphite is such that it shrinks to a certain 575 point and then expands again. This means extrapolation of the data into areas of extreme shrinkage (beyond the turnaround point) is unlikely and is, 530 to date, unobserved in the station data.

\section{Generalized Model}

The existing and new bore estimation models discussed in this paper to this point have only been trained on data from a single reactor, and with the most recent improvements, are approaching the quoted accuracy of $\pm 1 \mathrm{~mm}$. However, there are three other reactors in the UK that have very similar designs and thus produce very similar FGLT data. In an attempt to produce a generalized model that can be used on all four of these reactors it was first necessary to produce a set of results for the remaining three reactors using the linear model trained in the previous section, as it was shown that this produced the best results for the original reactor.

There are 50, 58 and 60 pairs of inspection and monitoring data available for the other reactors, as before half was set aside for testing while the other half was used for training. Using the selected testing sets, a bore estimation was produced for each channel for each station in the testing set. Then using the same metrics as used previously, results were produced for brick layers 6 to 11. Due to pausing the fuel movement to perform brush inspections during some refuelling operations, which coincides with the data in brick layer 5 , it was not possible to perform the estimation for the other stations below brick layer 6. Fig. 16 show the results produced, while the results for brick layer 6 are similar for all four stations. For brick layers 7 to 11, it is clear that the model does not accurately predict the bore diameter for the second station (reactors 3 and 4). It should also be noted that as expected both reactors 1 and 2 have very similar results as they are from the same station.

Having shown that the model trained only on data from one reactor is not an accurate model for the FGLT data from the second station (reactors 3 and 4), using the same approach as used previously a linear regression model was produced from the data of all four stations (109 FGLTs). The idea is that the increase in the amount of data used for training should produce a more accurate bore estimation model for all four stations than a model trained for any specific station. 


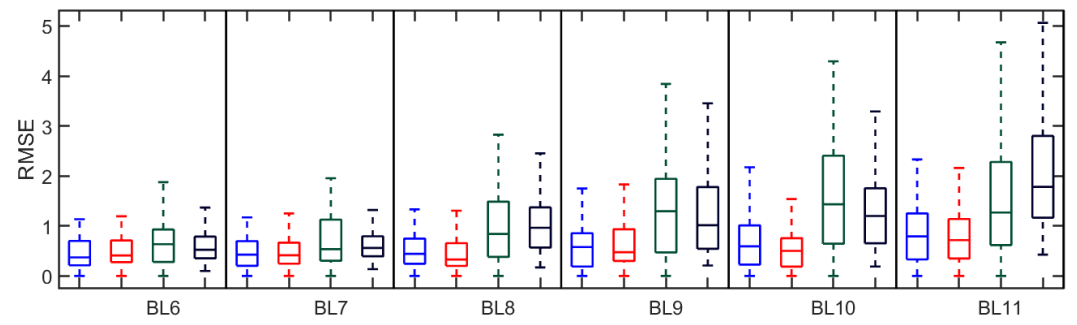

(a) Root mean square error for brick layer 6 to 11 in the test dataset

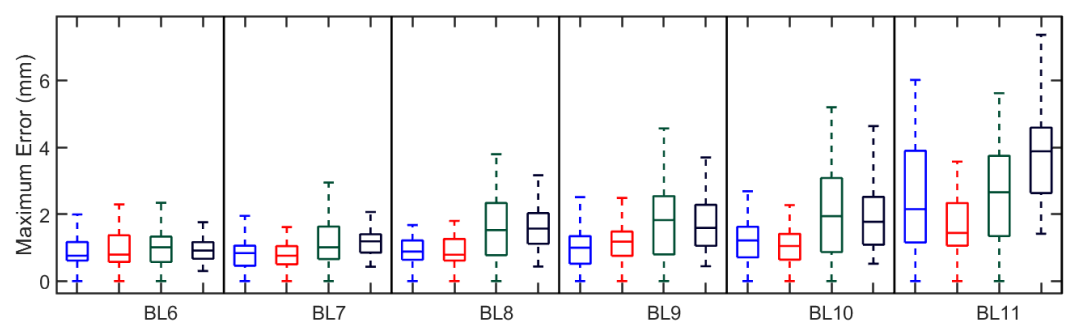

(b) Maximum error (mm) for brick layer 6 to 11 in the test dataset

Figure 16: Results for model V1.2, (Blue) Reactor 1, (Red) Reactor 2, (Green) Reactor 3, and (Black) Reactor 4

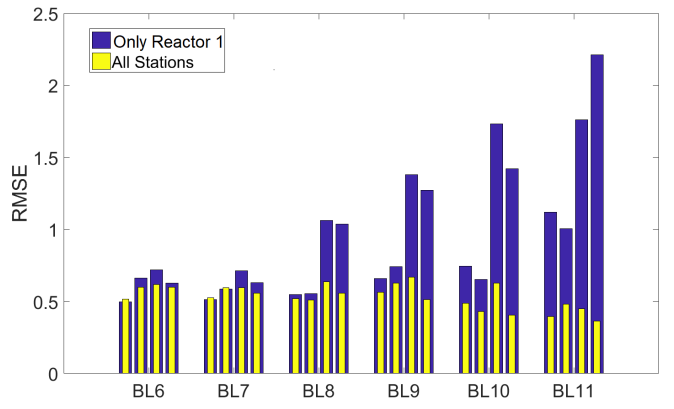

Figure 17: Average root mean square error for both models

Fig. 17 and 18 shows the results for both the RMSE and maximum error for both models on all four stations. It can be gathered from the results that there is very little change in the results for brick layer 6 , this is likely to be due to the fact that the model was already producing acceptable estimations. For brick layers 7 to 9 the estimation for station 2 is now on a level with the station 1 estimations and for brick layers 10 and 11 there is

\section{Conclusions and Future Work}

This paper has presented a generalised model for estimating fuel channel bore from FGLT data of

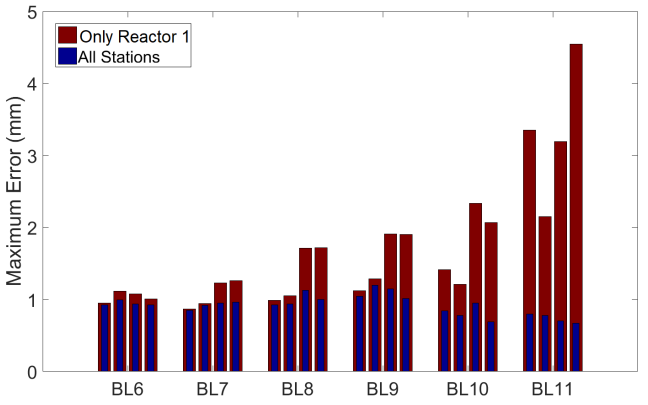

Figure 18: Average maximum error ( $\mathrm{mm}$ ) for both models

multiple advanced gas-cooled reactor cores. Firstly, 590 various implementation improvements were described that improved the accuracy of the existing bore estimation model, as well as retraining the existing linear regression model. Based on the literature a quadratic and cubic model was also trained. All these models were extensively tested with 25 FGLTs from the original reactor and it was found that while the quadratic and cubic model did provide better estimates in brick layers 5 to 9 , at the extremes where there was little or no training data the model fell down due to its non-monotonic nature. Having selected a linear model as the optimal choice for the original reactor, a generalized model was created to provide bore estimation for three ad- 
ditional reactors. This generalized model produced 660 mproved results for all stations and all brick layers due to the fourfold increase in training data with a decrease in an average maximum error of almost $80 \%$.

\section{Acknowledgement}

The authors would like to thank EDF Energy for providing the data used in this paper and the technical expertise. This work was funded by EDF Energy, however, the views presented by the authors do not represent the views of EDF Energy.

\section{References}

Berry C, Pattison D, West G, McArthur S, Rudge A. Estimating fuel channel bore from fuel grab load trace data. In: The 5th EDF Energy Nuclear Graphite Symposium. Warrington. 2017. p. 360-9.

. radiated graphite properties. Journal of Nuclear Materials 2008;381:137-44. doi/https://doi.org/10.1016/j. jnucmat.2008.07.040

British Electricity International . Modern Power Station Practice. Pergamon, 1992.

Brocklehurst JE, Kelly BT. Analysis of the dimensional changes and structural-changes in polycrystalline graphite under fast-neutron irradiation. Carbon 1993;31:155-78. doi 10.1016/0008-6223(93)90169-b

Cole-Baker A, Reed J. Measurement of AGR graphite fuel brick shrinkage and channel distortion. In: Neighbour G, editor. Management of Ageing Processes in Graphite Reactor Cores. Cambridge: RSC Publishing; 2007. p. 201-8.

Crump T, Fert G, Mummery P, Jivkov A, Martinuzzi P Tran VX. Dynamic fracture effects on remote stress amplification in agr graphite bricks. Nuclear Engineering and

1] Design 2017;323:280 -9. doi https://doi.org/10.1016/ j.nucengdes.2017.01.014

Marsden BJ, Hall GN, Wouters O, Vreeling J, van der Laan J. Dimensional and material property changes to irradiated gilsocarbon graphite irradiated between 650 and $750^{\circ} \mathrm{C}$. Journal of Nuclear Materials 2008;381:62-7. doi https ://doi.org/10.1016/j.jnucmat .2008.07.018

Maul P, Robinson P, Burrow J. Statistical modelling of 705 graphite brick weight loss in advanced gas cooled reactors. Nuclear Engineering and Design 2017;323:156 -65. doi https://doi.org/10.1016/j.nucengdes.2017. 08.017

Pang Y, Giovanini L, Monari M, Grimble M. Condition monitoring of an advanced gas-cooled nuclear reactor 7 core. Proceedings of the Institution of Mechanical Engineers, Part I: Journal of Systems and Control Engineer-

ing 2007;221(6):833-43. URL: https://strathprints. strath.ac.uk/11904/

Skelton J. Station core monitoring - Experimental work to relate HPB/HNB grab trip load to bore shrinkage. Technical Report; Amec NNC; 2007.

Steer AG. AGR core design, operation and safety functions. In: Neighbour G, editor. Management of Ageing Processes in Graphite Reactor Cores. Cambridge: RSC Publishing; 2007. p. 11-8.

West G, Jahn G, McArthur S, McDonald J, Reed J. Data mining reactor fuel grab load trace data to support nuclear core condition monitoring. IEEE Transactions on Nuclear Science 2006;53:1494-503. doi 10.1109/TNS.2006.874725

West G, Jahn G, McArthur S, Reed J. Graphite core condition monitoring through intelligent analysis of fuel grab load trace data. In: Neighbour G, editor. Management of Ageing Processes in Graphite Reactor Cores. Cambridge: RSC Publishing; 2007. p. 232-9.

West G, McArthur S, Towle D. BETA: A system for automated intelligent analysis of fuel grab load trace data for graphite core condition monitoring. In: Neighbour G, editor. Securing the Safe Performance of Graphite Reactor Cores. Cambridge: RSC Publishing; 2009. p. 79-87.

West G, Wallace C, McArthur S. Combining models of behavior with operational data to provide enhanced condition monitoring of agr cores. Nuclear Engineering and Design 2014;272:11-8. doi $10.1016 / j$.nucengdes.2013.12. 067

World Nuclear Association. Nuclear power in the United Kingdom. http://www.world-nuclear. org/information-library/country-profiles/

countries-t-z/united-kingdom.aspx 2017. Accessed: 2017-12-19.

Young A, Aylward W, Murray P, West G, McArthur S, Rudge A. Fuel channel bore estimation for onload pressurised fuel grab load trace data. In: 6th EDF Energy Nuclear Graphite Conference. 2018. p. 8. URL: https://strathprints.strath.ac.uk/66469/

Dr. Andrew Young received the M.Eng degree (with distinction) in electrical and mechanical engineering and Ph.D. degree from the University of Strathclyde, Glasgow, U.K, in 2013 and 2017, respectively. His research interests include condition monitoring, data analytics and signal and image processing

Craig Berry received the M.Eng degree in electronic and electrical engineering from the University of Strathclyde, Glasgow, U.K., in 2014. He is currently pursuing a Ph.D. degree at the University of Strathclyde within the Institute for Energy and Environment where his Ph.D. is fully funded by the Duncan Hawthorne Scholarship. His research interests include machine learning, condition monitoring and data analytics.

Dr. Graeme West received the B.Eng. (Hons.) and Ph.D. degrees from the University of Strathclyde, Glasgow, U.K., in 1998 and 2002, respectively. He is a Lecturer in the Department of Electronic and Electrical Engineering at the University of Strathclyde. His research interests include intelligent system applications in condition monitoring, diagnostics and prognostics, particularly applied to the nuclear power generation industry.

Prof. Stephen McArthur received the B.Eng. (Hons.) and Ph.D. degrees from the University of 
Strathclyde, Glasgow, U.K., in 1992 and 1996, respectively. He is co-Director of the Institute for En720 ergy and Environment at the University of Strathclyde and Academic Director of its Advanced Nuclear Research Centre. His research interests include data analytics and distributed intelligence for energy system applications. 